\title{
LA METACOMUNICACIÓN: UNA APROXIMACIÓN A SUS CUALIDADES PARA LA TOMA DE DECISIONES DEL INGENIERO INDUSTRIAL
}

\section{The metacommunication: an approach to their qualities for the taking of decisions of the industrial engineer}

iD Isabel Ríos Barrios ${ }^{1}$

iD Juan Carlos Álvarez Yero ${ }^{1}$

(iD) Daima Thabet Cabrera'

'Universidad de Camagüey, Cuba.

Correspondencia:

M. Sc. Isabel Rios Barrios

isabel.rios@reduc.edu.cu

\section{RESUMEN}

El trabajo tiene el propósito de fundamentar las cualidades metacognitivas, semióticas y pragmáticas de la comunicación para la toma de decisiones. Se develan las carencias que en este sentido mantienen algunos enfoques relacionados con la comunicación. Los aspectos tratados constituyen un acercamiento a la metacomunicación desde los fundamentos conceptuales que se proponen, su estructura y componentes. Se establecen nuevas relaciones a partir del análisis de estos componentes expresado en síntesis a través de un flujograma que facilita su comprensión. Finalmente, se advierte la necesidad e importancia de la temática en la formación del ingeniero industrial, a través del análisis de la influencia que ejerce en ellos el comportamiento oportuno en el proceso interactivo que posibilitan una mayor eficacia en la estrategia empresarial y por ende una mayor contribución a la competitividad en la empresa.

Palabras clave: Metacomunicación, toma de decisiones, cualidades, Ingeniero Industrial.

\section{ABSTRACT}

The work has the purpose of basing the qualities metacognitive, semiotics and pragmatic of the communication for the taking of decisions. It is shown the lacks that this sense maintains some focuses related with the communication. The treated aspects constitute an approach to the metacommunication from the conceptual foundations that intend, their structure and components. Finally, it is noticed the necessity and importance of the thematic one in the industrial engineer's formation through the analysis of the influence that exercises in them the opportune behavior in the interactive process that you/they facilitate a bigger effectiveness in the managerial strategy and as consequence bigger contribution to the competitiveness in the company.

Key words: Metacommunication, taking of decisions, qualities, Industrial Engineer. 


\section{INTRODUCCIÓN}

El desarrollo actual, caracterizado por el impetuoso avance de la ciencia y la técnica; así como, la ocurrencia de grandes cambios y transformaciones en el contexto académico, requiere la búsqueda de nuevas concepciones que permitan obtener resultados cualitativamente superiores, para el desempeño exitoso de los futuros egresados en las diferentes esferas de su actividad laboral.

En este sentido, la universidad tiene entre sus principales concepciones "(...) brindar a la sociedad un profesional formado de manera íntegra, profesionalmente competente, con preparación científica para aceptar los retos de la sociedad moderna (...)" (Horruitinier, 2009, pág. 26), con el fin de afrontar de manera independiente y creadora la realidad en las diferentes entidades laborales, productivas y de servicios a lo largo y ancho del país.

Estas condiciones precisan la formación de competencias profesionales en los egresados, no solo en términos del sistema de conocimientos y habilidades de las diversas disciplinas y asignaturas, sino que se ha de tener en cuenta la sensibilidad social, la innovación para vencer obstáculos, tanto externos como internos, el protagonismo en la dirección de los diferentes procesos, el trabajo en equipo, el enseñar a otros, el servicio con calidad a los clientes, la toma de decisiones para enfrentar posibles soluciones al hacer frente a las incertidumbres, así como demostrar interés y disposición por el crecimiento personal.

Una de las vías ineludibles para lograr éxitos en estos procesos psicológicos lo constituye el desarrollo de la metacomunicación, la cual genera mayor eficacia en la estrategia empresarial y por ende una mayor contribución a la competitividad en la empresa. La exploración en los elementos informativos refuerza la correcta toma de decisiones, la compatibilidad entre el mensaje y los trabajadores, la motivación por una mayor calidad comunicativa para sustentar la permanencia del proceso y la creación de una atmósfera de comprensión empática, de estimación y aceptación incondicional, respeto y compromiso.

Por otro lado, la metacomunicación se convierte en una herramienta de beneficio a la empresa, un buen sistema de mediación entre la codificación y la descodificación; ofrece recursos necesarios para resolver la gestión del cambio, cómo manejarlos y cómo reducirlos. Es por ello, que su uso provee el continuo seguimiento y orientación de los avances de metas, revisión de fortalezas, debilidades, desafíos y proyecciones.

En los últimos años se manifiesta una creciente preocupación sobre el estudio de diferentes aspectos de la metacomunicación, referidas a su concepción y estructura, evidenciada en los trabajos de numerosos investigadores cubanos y foráneos, entre ellos Zabalza, 2007; Ongallo C, 2007; Enrique, 2007; Prado C, 2012; Mendoza J.A, 2014. Sin embargo, no se ha profundizado los suficiente en otros componentes esenciales relacionados con la retroalimentación o feed back del proceso, que permita la reformulación y reducción de barreras durante el acto comunicativo, manifestados a través de la escucha defectuosa, desconfianzas en la comunicación, exceso de información, percepción selectiva, actitud predeterminada y una mala selección del contexto o entorno lingüístico y circunstancias que puedan afectar a los individuos que intervienen al establecerla.

Desde esta perspectiva se descuida el rol que juega la calidad de la retroalimentación en el éxito comunicativo, la inapropiada evaluación de las características del perceptor, la limitación de la capacidad para discriminar las informaciones, la carencia en la claridad del mensaje al comunicarse con códigos distintos; así como, la falsa interpretación a partir de estereotipos.

Tomar conciencia de la importancia que posee el significado de las impresiones mutuas, que conlleven a un estado emocional que permita la comprensión entre los sujetos como expresión perceptiva de los nexos comunicativos y del modo en que se establecen, es el propósito 
fundamental de este trabajo, asumir el estudio de la comunicación desde propiedades poco tratadas, que permitirán mejorar la comunicación partir de develar las relaciones semióticas, metacognitivas y pragmáticas como propiedades metacomunicativas.

\section{DESARROLLO}

La comunicación al ser una categoría polisemántica genera polémica desde las diferentes ciencias. Su origen radica en su propia etimología, communicare o communis, la cual expresa compartir o hacer común. Es por ello que, el análisis de las fuentes teóricas corrobora la existencia de numerosas definiciones del término, a partir de disímiles posiciones y del énfasis en unos $u$ otros aspectos del proceso comunicativo.

Aunque no es posible hallar unanimidad en este sentido es significativo destacar referencias comunes que sirve de orientación teórica metodológica para su estudio y caracterización. Desde las definiciones se plantean que es un proceso que devela su carácter participativo, continuo, dinámico, transformador, sistémico entre los sujetos (s-s). Se utilizan palabras, gestos y símbolos (comunicación verbal y no verbal). Se implica la personalidad como un todo. Se sustenta sobre la base de metas, fines u objetivos. Implica influencia mutua colaboración, cooperación y crecimiento.

Muchos son los que dirigen sus estudios a lo antes mencionado, Leontiev 1986; González 1995; Báxter 1999; Fernández 2002; pero es válido destacar que existen elementos que aún no quedan explícitamente detallados y conllevan a una toma de decisiones incorrecta.

Como proceso interactivo, la comunicación está sujeta a modificaciones dado por el dinamismo de las influencias mutuas de los participantes que definen sus relaciones dotándola no solo de un carácter interaccionar sino también transaccional, o sea, concertado por las partes de manera voluntaria con el fin de evitar las divergencias de significados.
Para el logro de lo antes expuesto se precisa que la comunicación transite por la distinción, saber interpretar, del tipo de mensaje que se percibe para poder dar una respuesta acertada, más allá de un intercambio "frío" de información que sólo tiene en cuenta el contenido del mensaje que se transmite y no la relación entre los participantes.

Existe la tendencia a creer que entender al otro y hacerle comprensible el mensaje, durante la comunicación, es algo sencillo, sin embargo, pudieran surgir dudas, malentendidos, desacuerdos y conflictos que resultarían limitantes sino se logra superar su efecto sobre el perceptor cuya reacción influirá a su vez sobre el emisor. Para la eficacia y calidad del proceso comunicativo se necesita de una relación entre la información (/o que se dice) y la retroalimentación ( /o que se capta de vue/ta) que esté mediada por actitudes reflexivas que permitan sostener la estabilidad de las relaciones.

Bueno y Garrido (2012), han declarado la importancia que tiene la retroalimentación en la comunicación como estrategia pueden interrumpir, detener o entorpecer el proceso comunicativo y dificultar la consecución de los objetivos. De modo para subsanar los errores y las barreras que impiden que el mensaje fluya adecuadamente, ya que estas circunstancias que, la efectividad de la comunicación adquiere un carácter reversible, circular, ya que permite modificar las sucesivas acciones comunicativas del emisor.

Por ello, este proceso no solo depende de la información que se pretende compartir, sino además de la forma en que es interpretada y evaluada por el perceptor, por ende, ignorar en qué medida se cumplió con la intención del mensaje evitaría entre otras cosas, la incoherencia y distorsión entre los interactuantes. Ciertamente la retroalimentación constituye la base y el comienzo de toda interacción, o sea, informar y compartir se sitúan en polos opuestos en una posible acción de comunicar puesto que el primero significa que una información pase a otro limitando lo transaccional de ella (la comunión, 
el encuentro, la participación y la elaboración de la información).

Es imprescindible aclarar que la retroalimentación puede estar limitada por varias causas: por el contenido de la información y por lo que se denota y connota en este proceso de interacción, es aquí donde toma toda su fuerza el significado de metacomunicación.

\section{¿Qué entendemos por metacomunicación?}

La palabra metacomunicación está compuesta por el (prefijo) "meta" entendido como "acerca de, entre, en medio de, después, de acuerdo con"y el término "comunicación" expuesta como proceso interactivo entre dos o más sujetos en el cual se comparten informaciones, emociones, sentimientos y vivencias.

El término metacomunicación tiene génesis en los axiomas de la comunicación humana estudiados por el psicólogo Watzlawick (1995), donde plantean que "Toda comunicación tiene un aspecto de contenido y un aspecto de relacional tales que el segundo clasifica al primero y es, por ende, una metacomunicación" (p. 12). Ver la figura 1.

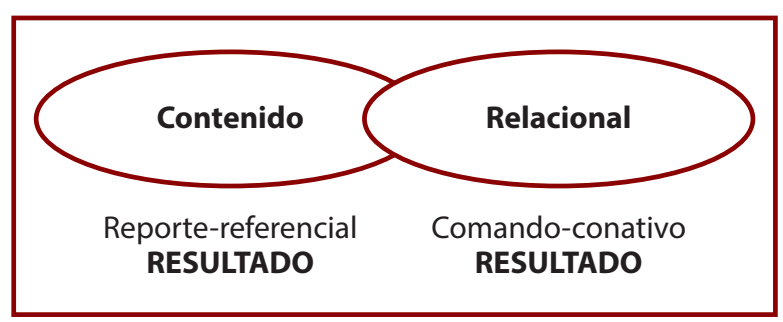

Figura 1. Contenido de la metacomunicación

Es evidente que para los sujetos que interactúan el entender lo que me están diciendo y el cómo debo interpretar el contenido en función de la relación que tengo con la otra persona son condiciones indispensables para mantener la relación con el otro. Lo antes planteado refleja que, en la comunicación, no solo está el contenido del mensaje, sino que también, existe una especie de mensajes ocultos que están determinados por la actitud o la manera de emitir el mensaje, que a su vez generará una actitud especifica en el interlocutor, a estos dos sentidos del mensaje se les podría llamar aspectos referenciales y connotativos.
Desde esta perspectiva se postula que toda comunicación dispone del contenido (referencial- "lo que se dice"-información) y de lo relacional (connotativos - "cómo se dice"). De hecho, para entender el contenido de una comunicación, se debe entender la relación de los comunicantes. De modo que, un mensaje no solo transmite información, lo que es sinónimo de contenido del mensaje, sino que a través de este se establece una relación entre los comunicantes Watzlawick, Beavin y Jackson (1981), lo que en última instancia se refiere al aspecto conativo.

Escartín y Suárez (2004), refieren que la metacomunicación "es el significado de lo que se dice o hace, la interpretación (...)". Es por ello que la significación de la comunicación depende de los sujetos implicados en ella, a su vez, las características de los sujetos determinan el proceso comunicativo". Es indudable en el proceso interactivo la necesidad de integrar lo referencial y lo relacional y su complemento está en ¿quién?, ¿con quién?, ¿cómo?, ¿cuándo?, ¿dónde?, ¿bajo qué circunstancias?, ¿qué tipo de relación? se manifiesta (p. 12).

Por otro lado, Satir (1986) plantea que es "la comunicación sobre la comunicación" (p. 3), es decir, aquella comunicación que habla acerca de la comunicación misma donde el interpretar y comprender lo que se dice o hace (referencial) en función del código empleado para ello (sistema de signos y de reglas que permite formular y comprender un mensaje) y de la relación entre los comunicadores (deseos, expectativas, intenciones y juicios valorativos).

En este proceso de decodificación de la información es importante que los sujetos adopten actitudes que conduzcan a resolver satisfactoriamente los problemas o conflictos que pudieran surgir en la interacción y que en ocasiones van acompañados por el aspecto emocional, motivacional y valorativo de los sujetos. En tanto, la metacomunicación es cuando dejamos de utilizar la comunicación para comunicarnos y la usamos para comunicar algo acerca de la comunicación. Así utilizamos conceptualizaciones que no son parte de 
la comunicación, sino que se refieren a ella (Watzlawicky, 1995).

En la retroalimentación la metacomunicación permite interiorizar en la relación en cuanto al mensaje, realiza ajustes en el momento y se orienta en las acciones sobre la base de la toma de decisiones, ofrecer información oportuna y positiva para aprender de errores y subsanarlos, así como identificar los aciertos y reforzarlos. No es general o abstracta sino específica y con base en los objetivos del curso que se está llevando a cabo, favorece la autoevaluación al recibir una crítica constructiva, aclara las metas y expectativas que se espera sean alcanzadas, refuerza las acciones y prácticas correctas, provee las bases para corregir errores.

En este caso, la metacomunicación trae consigo la manifestación de actitudes que demandan de la comprensión y aceptación del otro, ponerse en sus puntos de vista, sus razones, sus sentimientos sobre la base de la bilateralidad, reciprocidad, la participación activa y la contribución recíproca lo que trae consigo provocar o no la cooperación, así como ayudar o atraer a los participantes y de este modo viabilizar el logro de un objetivo común, conforme a la influencia mutua que ejerce uno sobre otro bajo el control de las actitudes.

En general, la metacomunicación da seguridad flexibilidad, perseverancia, iniciativa, confianza, autocontrol, cooperación, compromiso con los demás, demanda superación de errores y/o insuficiencias, exige tener en cuenta a los otros, donde se exprese lo que se piensa, quiere, siente y el por qué se ha tomado una decisión sobre la base del respeto mutuo, la nula mal sanidad, en fin, devenido en un proceso de comunicación dirigido a la ganancia y logros de fines comunes. Busca asegurar la eficacia del proceso comunicativo de forma bidireccional, es decir verificar, la forma en que el destino recibe el mensaje y su apropiación. Juega un papel muy importante en la comunicación porque permite al emisor conocer cómo se están interpretando sus mensajes y la ocurrencia de las fallas.
Se distinguen en este proceso la metacomunicación implícita y explícita.

- "La metacomunicación implícita de un mensaje es el modo en que éste es comunicado. A su vez, el modo en que se comunica algo metacomunica acerca de la relación existente entre emisor y receptor y marca las pautas en la interacción. Ese modo en que algo es comunicado comprende también la gestualidad propia de todo acto comunicativo.

- La metacomunicación explícita se refiere a la necesidad de pedir explicaciones acerca de aquellos mensajes confusos o contradictorios. En este caso, se dice que la metacomunicación es explícita porque los propios participantes del acto comunicativo son conscientes de que algo no funciona e intercambian información acerca de ello".

La metacomunicación se convierte en una herramienta eficaz que permite:

- Obtener información acerca de cómo es la relación afectiva entre los sujetos. La forma o modo de decir permite que los sujetos alcancen sus metas más rápidas y satisfactoriamente. Este proceso se convierte en la fuente de enriquecimiento afectivo donde se evidencian sentimientos, valores, convicciones, ideales.

- Reflexionar sobre la efectividad de la información decodificada a partir del conocimiento que tenga el sujeto de sus fortalezas y debilidades, el control y evaluación en el proceso (diagnóstico). Si una persona tiene conocimiento de sus procesos psicológicos propios, podrá usarlos más eficaz y flexiblemente en la planificación estrategias para comunicarse.

- Organizar estrategias comunicativas que permitan el 'vernos' en la relación, el 'chequear' cómo nos está 'yendo' donde se fomenten actitudes de colaboración, compatibilidad, ayuda, satisfacción y acercamiento que incidan en un adecuado clima socio psicológico. 


\section{¿Qué entender por entropía comunicativa?}

Entropía es una palabra que procede del griego (Évtpottía) que significa "evolución" o "trasformación". Sus orígenes datan de 1858 por el físico alemán Rudolf J. E. Clausius donde lo acuña como unidad de medida del desorden de un sistema. Cuando se dice que aumentó la entropía en un sistema, significa que creció el desorden en ese sistema. Y a la inversa: si en un sistema disminuyó la entropía, significa que disminuyó su desorden.

El concepto de entropía es diverso, su existencia está vinculada con diversas ciencias como la termodinámica, mecánica, estadística y teoría de la información. El concepto básico de entropía en teoría de la información tiene mucho que ver con la incertidumbre que existe en cualquier experimento o señal aleatoria. Es también la cantidad de "ruido" o "desorden" que contiene o libera un sistema.

La entropía aparece cuando la comunicación no fluye, o sea, la información que se codifica se distorsiona e impide que se produzca la retroalimentación. En este sentido el proceso comunicativo se "degrada" y no se logran cambios positivos, no habrá entendimiento, ni se compartirán los mensajes, a partir de entonces ocurrirá la aparición de conflictos o problemas.

A tono con lo anteriormente expuesto, el ruido desde la teoría de la información se declara como todo lo que altera el mensaje e impide que este llegue correcto y fielmente al destinatario; todo lo que perturba la comunicación, la obstaculiza, la interfiere o la distorsiona.

En consecuencia, con todo lo anteriormente expuesto, se puede afirmar que existen factores que bloquean o interrumpen el proceso comunicativo, los cuales se denominan barreras o ruidos en la comunicación. Estos conllevan a un aumento de la entropía comunicativa, lo que a su vez degrada la comunicación y no se lograrán cambios positivos, no habrá entendimiento, ni se logrará empatía en los mensajes. A partir de entonces no ocurrirá nada ordenado, para restaurar el orden y lograr la reversibilidad de la situación, hace falta un esfuerzo especial de los sujetos que intervienen.

Sin dudas en todo proceso metacomunicativo de carácter formativo suelen ponerse de manifiesto diferentes factores que intervienen en la calidad del proceso, entre los cuales están:

- Lo personológico: significa que los sujetos que interactúan expresan su sello de su individualidad. Esto refiere aprovechar de manera efectiva sus recursos metacognitivos para la dirección y control de la interacción. Si en esta dinámica el sujeto regula y autorregula su actitud comunicativa, el proceso se convierte en un lugar de cambio y transformación, es decir, hacia un crecimiento personal y social.

- La significación de la información, la comprensión de lo que transmite el mensaje y su utilidad. Esto implica por parte de la semiótica una interpretación a partir de los signos según su naturaleza (tipo y tono) y su relación con los objetos dinámicos (ícono, Índice y símbolo) y funciones (emotiva, referencial, fática y metalingüística).

- El contexto y momento, o sea, dónde se establece la comunicación. Desde la pragmática en el contexto comunicativo interviene la situación (lugar y tiempo). Por otro lado, está el momento en que se comparte la información.

De esta manera, al valorar la metacomunicación como proceso que regula y autorregula el intercambio de información entre los participantes, donde un sujeto produce y evalúa la información que transmite a partir de sus características personológicas y el otro la descodifica interpretando y valorando lo que le llega y viceversa.

Desde esta dirección el contenido del mensaje puede ser valorada de forma positiva o sea cuando existe compatibilidad entre objetivoresultado o de forma negativa cuando aparece la entropía entre el objetivo-resultado.

Ver la figura 2. 


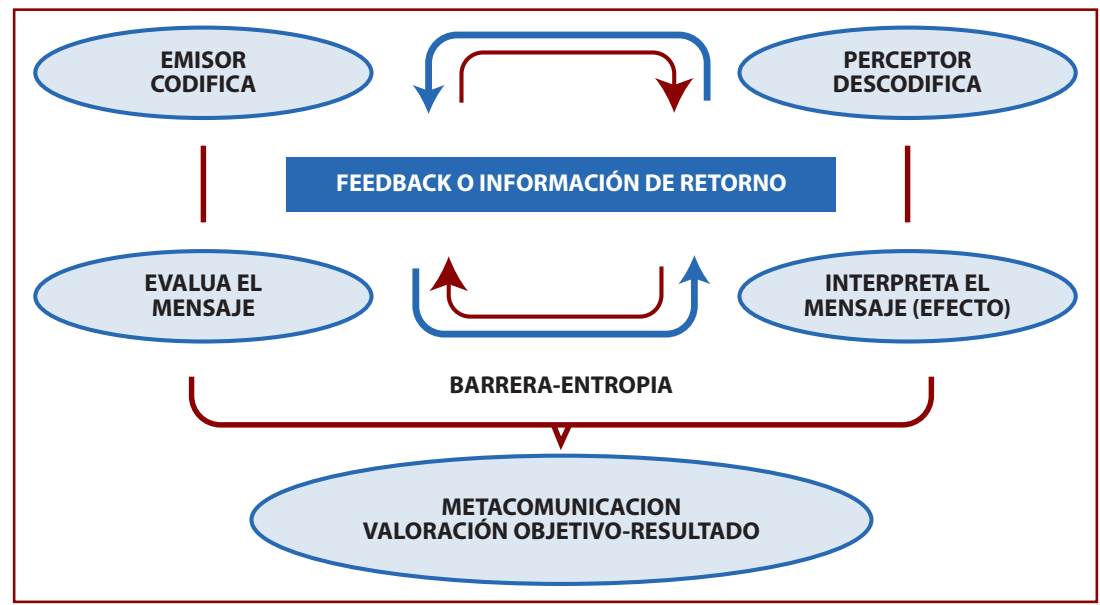

Figura 2. El proceso metacomunicativo.

Por su parte, solo a través de la metacomunicación se logrará establecer el modo para el esclarecimiento de aspectos no entendidos o dudosos en principio para que los interlocutores ganen información disminuyendo así la entropía y por ende las probabilidades de desorden. De esta manera, se aumentan las posibilidades de certeza para la toma de decisiones, sobre todo para las que se toman en grupo, pues la mayoría de estas serán consensuadas si se forman a partir del entendimiento mutuo, lográndose un proceso mucho más trasparente desde lo decisorio.
De modo que, el proceso metacomunicativo para la toma de decisiones consensuadas puede representarse a partir del siguiente flujograma. Nótese que, cuando el perceptor descodifica el mensaje o la información según los fines del emisor, la toma de decisiones consensuadas transita de manera coherente y fluida. En caso de que el mensaje no se descodifica por parte del perceptor, en correspondencia con el objetivo del emisor, aparece la entropía comunicativa y por ende se necesita acudir a la metacomunicación para gestionar una toma de decisiones consensuadas. Ver la Figura 3.

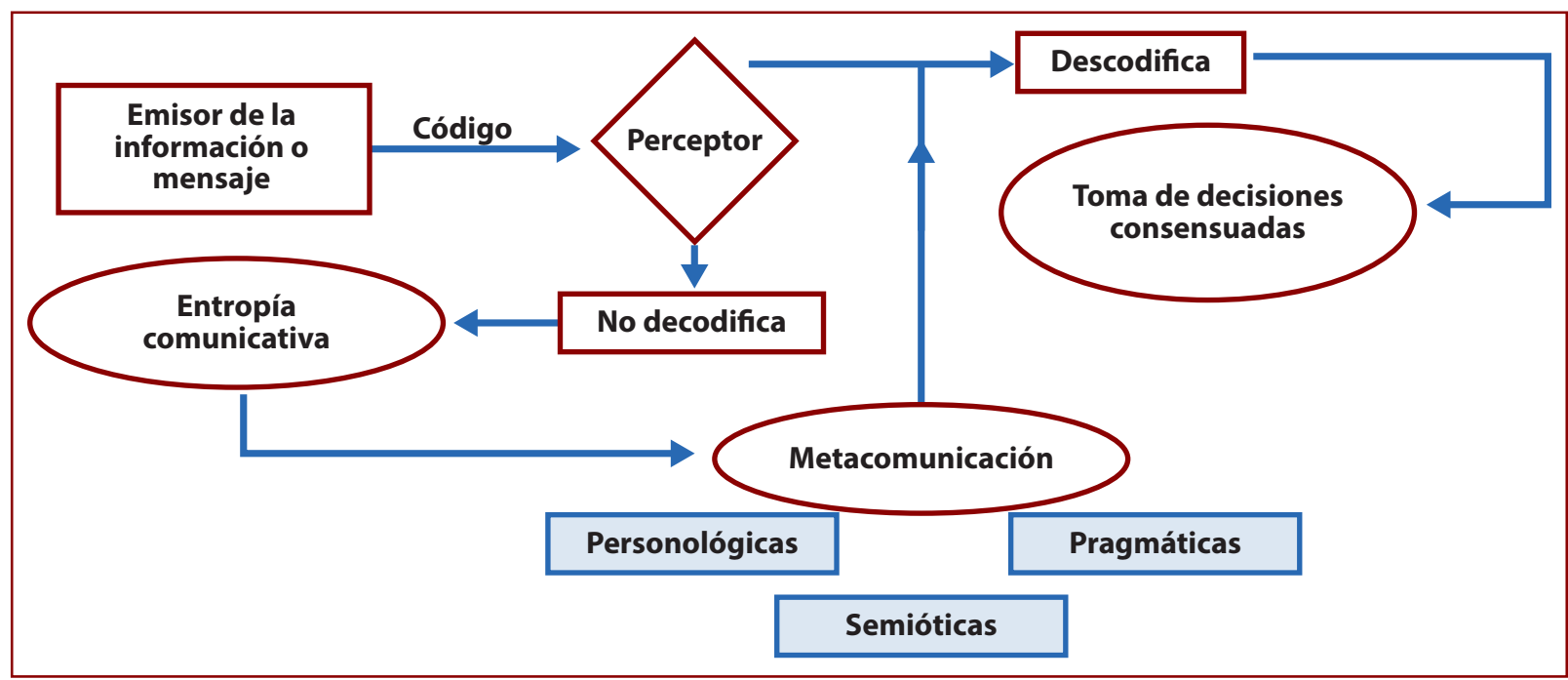

Figura 3. Flujograma-modelo del proceso metacomunicativo para la toma de decisiones. 
Aun así, es difícil eliminar las incertidumbres que generan la entropía comunicativa, no obstante, en la medida que se logre minimizar los ruidos o barrerascausantesaumentarán las probabilidades de que la comunicación sea fluida, directa y bidireccional. Estas cualidades propiciarán que la toma de decisiones sea generalmente de común acuerdo, lo que aumenta las posibilidades de lograr el efecto deseado.

Este proceso fue incluido en asignaturas del currículo propio y optativo de la carrera de Ingeniería Industrial de la Universidad de Camagüey, durante el estudio de los métodos de decisiones bajo conflicto, así como en las herramientas para la toma de decisiones. Asimismo, fue implementado desde la práctica laboral de dichos profesionales en formación. Su efectividad fue comprobada mediante la observación de sesiones de discusión en grupos en los que se buscaba llegar a la toma de decisiones sobre determinados problemas propios del ámbito empresarial.

En la tabla siguiente se resumen los resultados de 11 sesiones observadas, en relación a la aplicación o no del flujograma-modelo y su posible influencia en el logro de la toma de decisiones consensuadas (objetivo).

Tabla 2

Resultados de aplicación de flujograma-modelo

\begin{tabular}{|c|c|c|c|c|}
\hline $\begin{array}{c}\text { ENTROPÍA } \\
\text { COMUNICATIVA }\end{array}$ & METACOMUNICACION & $\begin{array}{c}\text { SE } \\
\text { ALCANZA } \\
\text { OBJETIVO }\end{array}$ & $\begin{array}{l}\text { SE ALCANZA } \\
\text { PARCIALMENTE } \\
\text { EL OBJETIVO }\end{array}$ & $\begin{array}{c}\text { NO SE } \\
\text { ALCANZA EL } \\
\text { OBJETIVO }\end{array}$ \\
\hline $\begin{array}{l}\text { La comunicación } \\
\text { no fluye, o sea, la } \\
\text { información que } \\
\text { se codifica se } \\
\text { distorsiona e } \\
\text { impide que se } \\
\text { produzca la } \\
\text { retroalimentación. }\end{array}$ & $\begin{array}{l}\text { Entonces interviene: } \\
\text { intercambio fluido, } \\
\text { ordenado, } \\
\text { entendimiento } \\
\text { mutuo, } \\
\text { retroalimentado, } \\
\text { consensuado. }\end{array}$ & $72.7 \%$ & $18.2 \%$ & $9.1 \%$ \\
\hline $\begin{array}{l}\text { La comunicación } \\
\text { no fluye, o sea, la } \\
\text { información que } \\
\text { se codifica se } \\
\text { distorsiona e } \\
\text { impide que se } \\
\text { produzca la } \\
\text { retroalimentación. }\end{array}$ & $\begin{array}{c}\text { Entonces no } \\
\text { interviene: se } \\
\text { degrada la } \\
\text { comunicación y no } \\
\text { se lograrán cambios } \\
\text { positivos, no habrá } \\
\text { entendimiento, ni se } \\
\text { logrará empatía en } \\
\text { los mensajes. }\end{array}$ & $27.2 \%$ & $36.4 \%$ & $36.4 \%$ \\
\hline
\end{tabular}

Es notable como se logró alcanzar el objetivo en una proporción 2.7 veces más cuando se aplica el flujograma-modelo que cuando este no interviene, aumentando así las posibilidades de éxito para la toma de decisiones consensuadas en grupo. También se evidencia en la no intervención un equilibrio entre las posibilidades de no alcanzar el objetivo y de alcanzarlo parcialmente. Esto precisaría del estudio de otras variables que pudiesen influir en estos resultados, que incluye en sí el análisis de por qué aún sin intervenir hay posibilidades de éxito, aunque sean mínimas.

\section{CONCLUSIONES}

Toda comunicación considera dos aspectos esenciales Ilamados referenciales y connotativos, el primero se refiere a su contenido, mientras que el segundo establece la eficacia del proceso, que implican la metacomunicación, ya que a 
través de este se establece la relación entre los comunicantes.

El mejoramiento del proceso comunicativo exige el develamiento y estudio profundo de la metacomunicación, poco atendida desde la teoría y evidenciadas desde la práctica.

La toma de decisiones consensuadas o de común acuerdo aumentará sus posibilidades de concreción en la medida que se logre disminuir la entropía comunicativa a través de la metacomunicación.

\section{REFERENCIAS BIBLIOGRAFICAS}

Arribas, U. (2000). Comunicación en la empresa. La importancia de la información interna en la empresa. Revista Latina de Comunicación Social, 3(27), Recuperado de http://www.ull. es/publicaciones/latina.

Alvarez, J.C. y Ríos, I. (2016). La formación de competencias informacionales en el ingeniero industrial. Revista Transformación, 12 (2), 42-52. Recuperado de https://revistas. reduc.edu.cu/index.php/transformacion/ article/view/1473

Águila, A (2006). Metodología para el desarrollo de la competencia comunicativa desde lo paralingüístico en estudiantes de la carrera de derecho. Tesis doctoral. Universidad de Camagüey "Ignacio Agramonte Loynaz", Camagüey, Cuba. Disponible en: http://rediuc.reduc.edu. cu/jspui/bitstream/123456789/1125/1/ AguilaCarralero_Alisvech.pdf

Báxter, E (1999). La comunicación educativa. ¿Le corresponde solo al maestro? La Habana: Editorial Pueblo y Educación.

Bendezú, R. (2001). Los dominios de la comunicación empresarial. Revista Pharos, 8 (1), Recuperado de http://www.redalyc.org/ articulo.oa

Gómez, M. (2007). La comunicación en las organizaciones para la mejora de la productividad: El uso de los medios como fuente informativa en empresas $e$ instituciones andaluzas. Tesis doctoral. Universidad de Málaga, España. Disponible en: http://www.biblioteca.uma.es/bbldoc/ tesisuma/17672697.pdf

González, A. (2010). Emociones desde una perspectiva psicobiológica. Monografía de licenciatura no publicada. Universidad Autónoma de Yucatán, Mérida, Yucatán, México.

Horruitinier, P (2009). La universidad cubana modelo de formación. La Habana, Cuba: Editorial Universitaria. Disponible en: https:// docplayer.es/97461281-La-universidadcubana-el-modelo-de-formacion-dr-pedrohorruitiner-silva.html

Jackson, Y. (1983). Teoría de la Comunicación Humana. Barcelona: Herder.

Jiménez, A. (2007). La comunicación empresarial en situación de crisis. Tesis doctoral. Universidad Autónoma de Barcelona, España. Disponible en: https://www.tdx.cat/ bitstream/handle/10803/4142/aej1 de1.pdf

López, L (2003). Comunicación Social. Editorial Félix Varela. La Habana, Cuba

López, S (2007). Metodología para el desarrollo de la competencia comunicativa, desde el componente paralingüístico, en la comunicación audiovisual de los estudiantes de la carrera de periodismo. Tesis doctoral. Universidad de Camagüey "Ignacio Agramonte Loynaz", Camagüey, Cuba

Ongallo, C. (2007). Manual de comunicación: Guía para gestionar el Conocimiento, la información y las relaciones humanas en empresas y organizaciones. Madrid, España: Editorial Dykinson. Disponible en: https:// www.comunicacioninterna.pe/pdf/pdf5. pdf 
Ministerio de Educación Superior (MES). (2019). Plan E para Ingeniería Industrial. La Habana, Cuba.

Ríos, I. y Alvarez, J.C (2016). La comunicación emocionalmente Inteligente en el entorno empresarial. Una mirada desde el ingeniero industrial. Ponencia en XIV Conferencia Internacional de Ciencias Económicas y Empresariales, evento en la Universidad de Camagüey "Ignacio Agramonte Loynaz", Camagüey, Cuba

Muchinsky, M. (2016). Psicología Aplicada al Trabajo, [en línea]. Recuperado de http:// www.insht.es

Watzlawick, P., Bavelas, B. y Jackson, D. (1995). Teoría de la Comunicación Humana. Barcelona: Herder. 